\title{
The morphological and physiological response of Lachenalia to supplemental irradiation
}

\author{
Anna Kapczyńska ${ }^{1}$ Bożena Stodolak ${ }^{2}$
}

Received: 13 November 2017 / Revised: 28 March 2019 / Accepted: 8 April 2019 / Published online: 8 July 2019

(c) The Author(s) 2019

\begin{abstract}
The aim of this study was to examine the effects of supplemental irradiation, provided by high-pressure sodium lamps during cultivation, on growth, flowering, and physiological responses of six Lachenalia cultivars: 'Rainbow Bells', 'Aqua Lady', 'Josephine', 'Riana', 'Radient', and 'Cherise'. Depending on light conditions and cultivar, lachenalia bulbs flowered from February 20th to March 24th. Additional irradiation accelerated flowering of 'Rainbow Bells', 'Aqua Lady', 'Josephine', and 'Riana' plants by 5-19 days depending on the cultivar. Compared with controls, the irradiated bulbs had a higher floral ratio and produced shorter inflorescence stems and inflorescences, a larger number of single florets, and thicker inflorescence stems (except for 'Rainbow Bells'). The use of lamps resulted in shorter leaves with a higher chlorophyll a content in 'Aqua Lady', 'Riana', 'Radient', and 'Cherise' compared to control plants. The shortest leaves were reported in the 'Josephine' cultivar, and they were on average two times shorter than in the other cultivars. Bulbs and leaves of all cultivars grown under supplemental irradiation were characterized by higher dry weight than control plants. Dry weight content was highest in the bulbs and in the roots, and it was lowest in lachenalia leaves and shoots. Thus, supplemental irradiation from high-pressure sodium lamps positively affected morphological and physiological plant quality, although differences in the evaluated features largely depended on cultivar.
\end{abstract}

Keywords Artificial light · Asparagaceae $\cdot$ Cape hyacinth $\cdot$ Flowering $\cdot$ Ornamental geophyte $\cdot$ Photosynthetic pigments

\section{Introduction}

Lachenalia is a bulbous ornamental plant of the Asparagaceae family. The genus comprises 133 endemic species occurring mainly in the winter rainfall zone of South Africa and Namibia (Duncan 2012). Several of them are listed in the Red Data Book of Endangered Species of South Africa (Kleynhans et al. 2012). Outside Africa, lachenalia has been known for over two centuries (Grace and Van Staden 2003), but it is still deemed a unique and special plant representing African flora. In the 1970s,

Communicated by So-Young Park, Ph.D.

Anna Kapczyńska

akapczynska@op.pl

1 Department of Ornamental Plants, University of Agriculture in Krakow, al. 29 Listopada 54, 31-425 Kraków, Poland

2 Department of Food Biotechnology, University of Agriculture in Krakow, ul. Balicka 122, 30-149 Kraków, Poland a comprehensive breeding program was initiated in the Republic of South Africa aimed at obtaining a series of new cultivars with flowers in different colors (Duncan 2012); as a result, lachenalia was registred under the trade name Cape Hyacinth (Kleynhans 2006). There are currently five commercially available cultivars (Duncan 2013), differing in color and flowering time. Most of these cultivars have spotted leaves and a long life span, and their inflorescence stems remain highly decorative for up to 3 weeks (Kapczyńska 2014). The course of growth and flowering of bulbous plants developing in their natural environment differs from that of the same plants grown under greenhouse conditions in other climatic zones (Halevy 1990). According to Kleynhans et al. (2002), one basic morphological criterion applied during the breeding process and commercialization of the resulting lachenalia cultivars was plant compactness. To achieve true-to-type and high-quality plants, future growers should breed the new cultivars based on detailed investigations of growth requirements and plant physiology. The timing of flowering is species or even cultivar specific, and it is strongly 


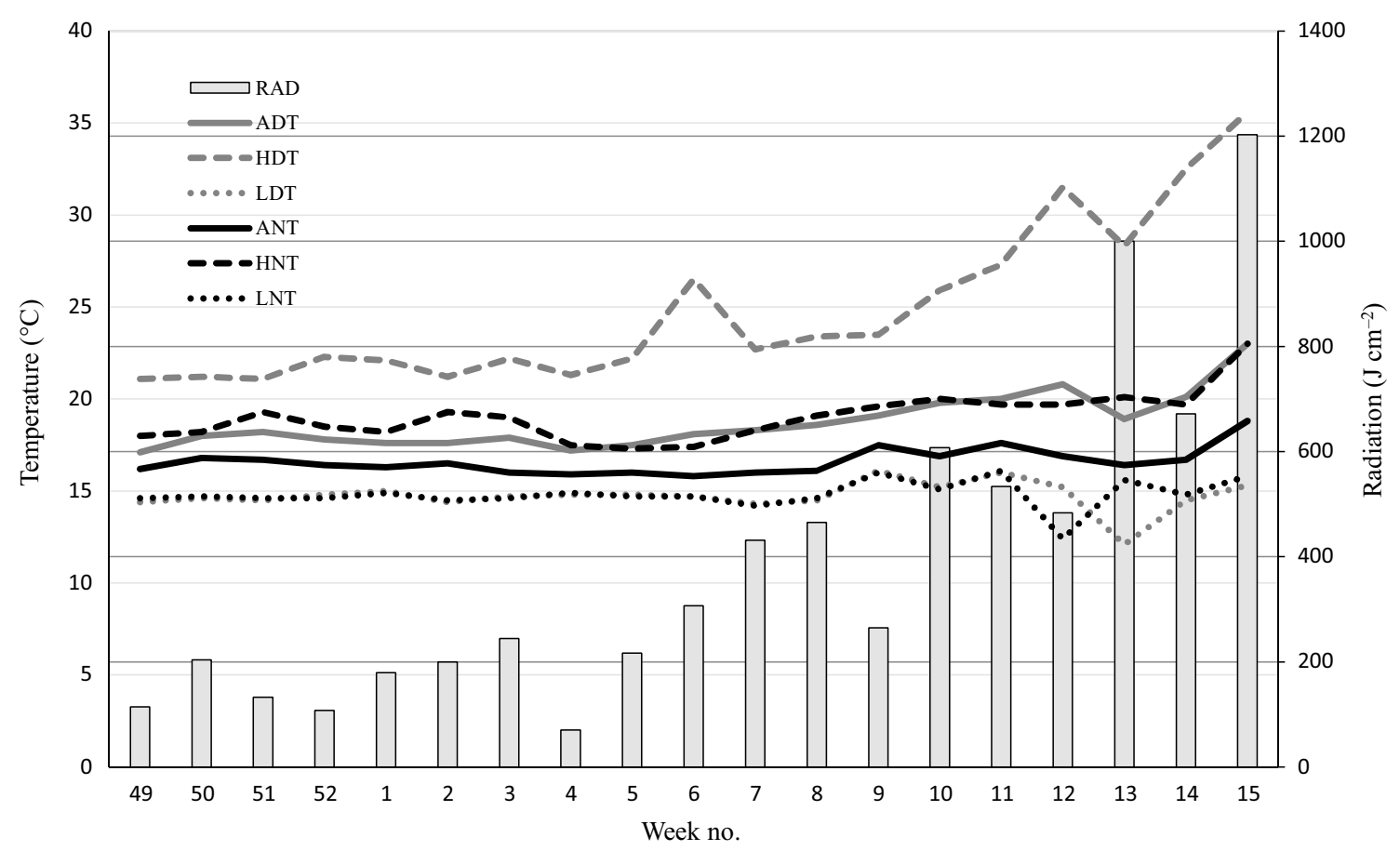

Fig. 1 Weekly average (A), lowest (L), highest (H), day (D), and night (N) temperatures (T) and radiation (RAD) during the experiment (from the 49th week of 2014 to the 15 th week of 2015)

affected by the environmental conditions of plant cultivation. Light duration, quality, and intensity play a crucial role in regulating the flowering process both in terms of time and quality (Thompson et al. 2011). In the northern hemisphere, low solar radiation in winter is a limiting factor for plant production. In such conditions, supplemental assimilation light could be used, as it improves plant quality and makes year-round cultivation possible (Heuvelink et al. 2006). Previous studies of supplemental light showed that increased irradiance played a crucial role in the development and flowering of many ornamental geophytes by increasing flower number per stem, decreasing stem length (Catley et al. 2002), by increasing stem and flower diameter (Lopéz et al. 2006), accelerating flowering (Han et al. 1991; Lopéz et al. 2006), or enlarging the percentage of flowering bulbs (Theron and Jacobs 1996). Moreover, a compact shape may be achieved by supplemental irradiation, which is a simple and nonpolluting method of controlling the growth of greenhouse-grown plants (Schwend et al. 2015; Bergstrand et al. 2016).

In this study, six new Lachenalia cultivars were exposed to supplemental lighting during a cultivation period in the greenhouse by high-pressure sodium lamps, and plant growth, yield, and inflorescence stem quality as well as physiological parameters of the irradiated and nonirradiated plants were investigated and compared.

\section{Materials and methods}

\subsection{Plant material and growth conditions}

The bulbs of six lachenalia (Lachenalia Jacq. ex Murray) cultivars, i.e., 'Rainbow Bells', 'Aqua Lady', 'Josephine', 'Riana', 'Radient', and 'Cherise', were purchased from Afriflowers (Cullinan, South Africa). The study was conducted in a glass-glazed Venlo greenhouse (equipped with Integro 724 computers; Priva) at the University of Agriculture in Krakow, Poland (lat. $50.08^{\circ} \mathrm{N}$, long. $19.95^{\circ} \mathrm{E}$ ). The greenhouse day/night heating temperature was set at $18 / 15^{\circ} \mathrm{C}$ (weekly average, lowest and highest day and night temperatures, as well as radiation during the experiment are presented in Fig. 1).

The bulbs were prepared for the experiment in the following temperature regimes during storage: $9{ }^{\circ} \mathrm{C}$ for 20 weeks, followed by 2 weeks at $35{ }^{\circ} \mathrm{C}$, and 22 weeks at $25{ }^{\circ} \mathrm{C}$. Immediately before planting, randomly selected bulbs were cut longitudinally through the middle of the basal plate to observe if the section revealed any first flower primordia, which would indicate that flowering induction had initiated (photographic documentation was prepared). On December 10,2014 , the bulbs that were approximately $2.0 \mathrm{~cm}$ in diameter and $5.0 \mathrm{~g}$ in weight were planted individually in standard round plastic pots $12 \mathrm{~cm}$ in diameter with a volume of $0.6 \mathrm{~L}$. Before planting, the bulbs were soaked in $0.25 \%$ 
Fig. 2 Spectral characteristics of the HPS lamp SON-T Agro (by M. Żupnik)

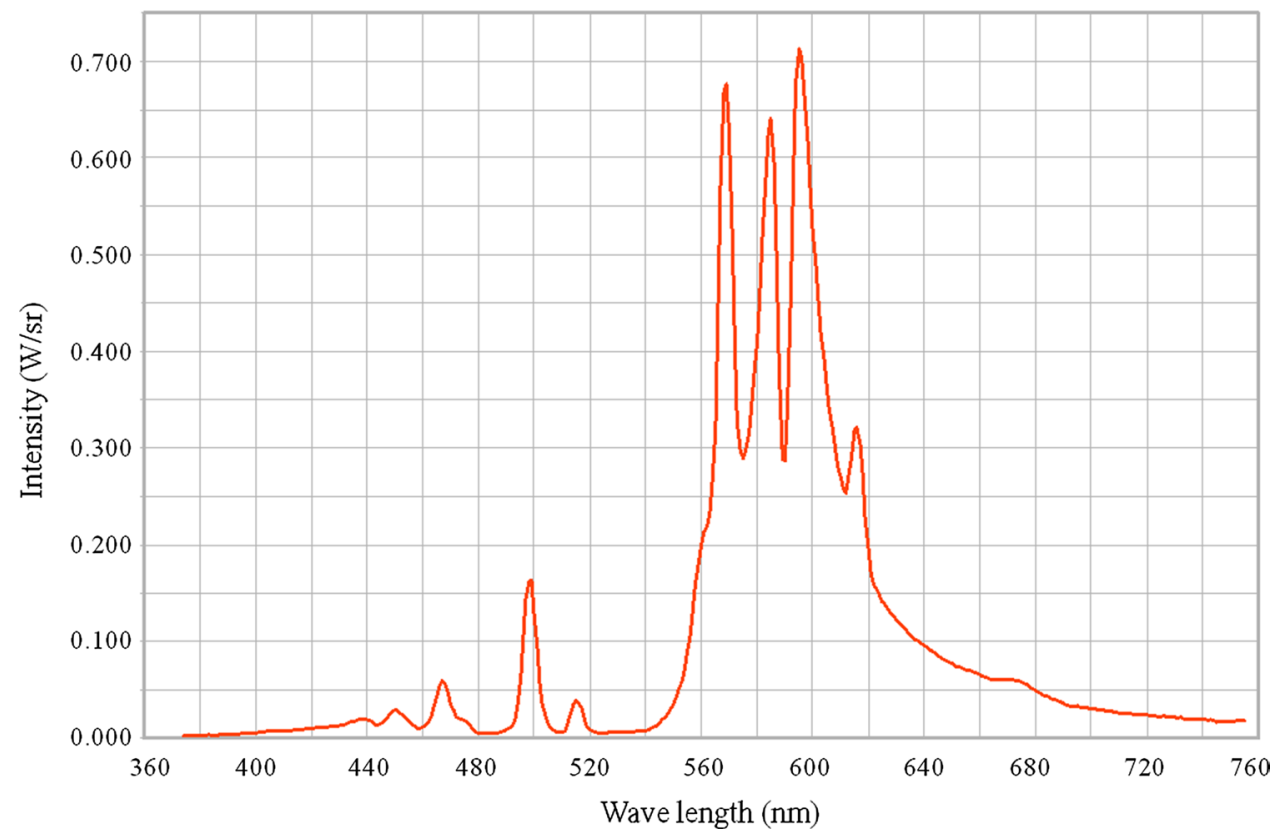

captan suspension for $30 \mathrm{~min}$. The pots were filled with a peat substrate (Botanica Professional Comeco, Poland). A chemical analysis of the substrate showed the following chemical composition $\left(\mathrm{mg} \mathrm{l}^{-1}\right), \mathrm{pH}=6.26: \mathrm{N}\left(\mathrm{N}-\mathrm{NO}_{3}\right), 112$; $\mathrm{P}, 36 ; \mathrm{K}, 282 ; \mathrm{Ca}, 1174 ; \mathrm{Mg}, 110 ; \mathrm{Cl}, 35$. The plants were irrigated manually and $100 \mathrm{ml}$ per pot of $30 \mathrm{~N}-0 \mathrm{P}-16.6 \mathrm{~K}$ liquid fertilizer with microelements (Florovit Universal; Inco-Veritas, Warsaw, Poland) at a concentration of $1.0 \%$ was applied every 2 weeks. Plants were randomly divided into two experimental groups per cultivar. The first group was grown only under natural light and served as controls (CTR). The second group also was grown under natural light but supplemental photosynthetic irradiation was additionally provided by 400-W high-pressure sodium (HPS) lamps (Philips SON-T Agro, USA) with a photon flux density (PFD) of $100 \pm 20 \mu \mathrm{mol} \mathrm{m}{ }^{-2} \mathrm{~s}^{-1}$ (measured during a cloudy day using the LI-250A Light Meter, LI-COR, Lincoln, Nebraska, USA). Supplemental irradiation (IR) was provided from 6.00 to $9.00 \mathrm{a} . \mathrm{m}$. and from 3.00 to $6.00 \mathrm{p} . \mathrm{m}$. during the entire cultivation cycle. Detailed spectral characteristics of the sodium lamps used in the study are presented in Fig. 2. For each cultivar, the experimental setup consisted of four replications of each light treatment (five bulbs per replication).

\subsection{Morphological parameters and time of flowering}

When the first floret opened (beginning of flowering), inflorescence stem height (from the substrate to the uppermost part of the inflorescence), inflorescence length, number of florets in the inflorescence, diameter of the inflorescence stem, length of a single floret (the first developed one), fullness ratio of the inflorescence (the quotient of the number of florets and inflorescence length), number of leaves produced by one bulb, and length and width (central part of the leaf) of the first and second leaves were recorded, together with the number of days from planting the bulbs to visible flower buds and to the beginning of flowering (anthesis).

\subsection{Determination of photosynthetic pigments}

Chlorophyll and carotenoid concentrations were determined spectrophotometrically (Spekol 1500, Analytik Jena, Germany) according to an equation provided by Arnon (1949). A 0.25 -g leaf sample was homogenized by grinding with $0.1 \mathrm{~g}$ of $\mathrm{CaCO}_{3}$ and $10 \mathrm{ml}$ of acetone in a glass mortar. The mixture was filtered through filter paper into a volumetric flask, and the sediment left on the filter paper was washed with acetone until the pigments were thoroughly removed. The extract was filled up to $25 \mathrm{ml}$ with acetone and then diluted with distilled water so that the final concentration of acetone was $80 \%$. Absorbance was read at $645 \mathrm{~nm}$ for chlorophyll b ( $\mathrm{Chl} \mathrm{b),} 663 \mathrm{~nm}$ for chlorophyll a (Chl a), and $440 \mathrm{~nm}$ for carotenoids (Car) against $80 \%$ acetone as blank. Photosynthetic pigment content was determined 2 months after planting the bulbs. Three analytical replications were performed for each treatment.

\subsection{Determination of dry weight}

For determination of dry weight, aboveground (leaves and inflorescence stems) and underground (bulbs and roots) parts were dried in a sterilizer (Sanyo Mov 112S, Japan) 


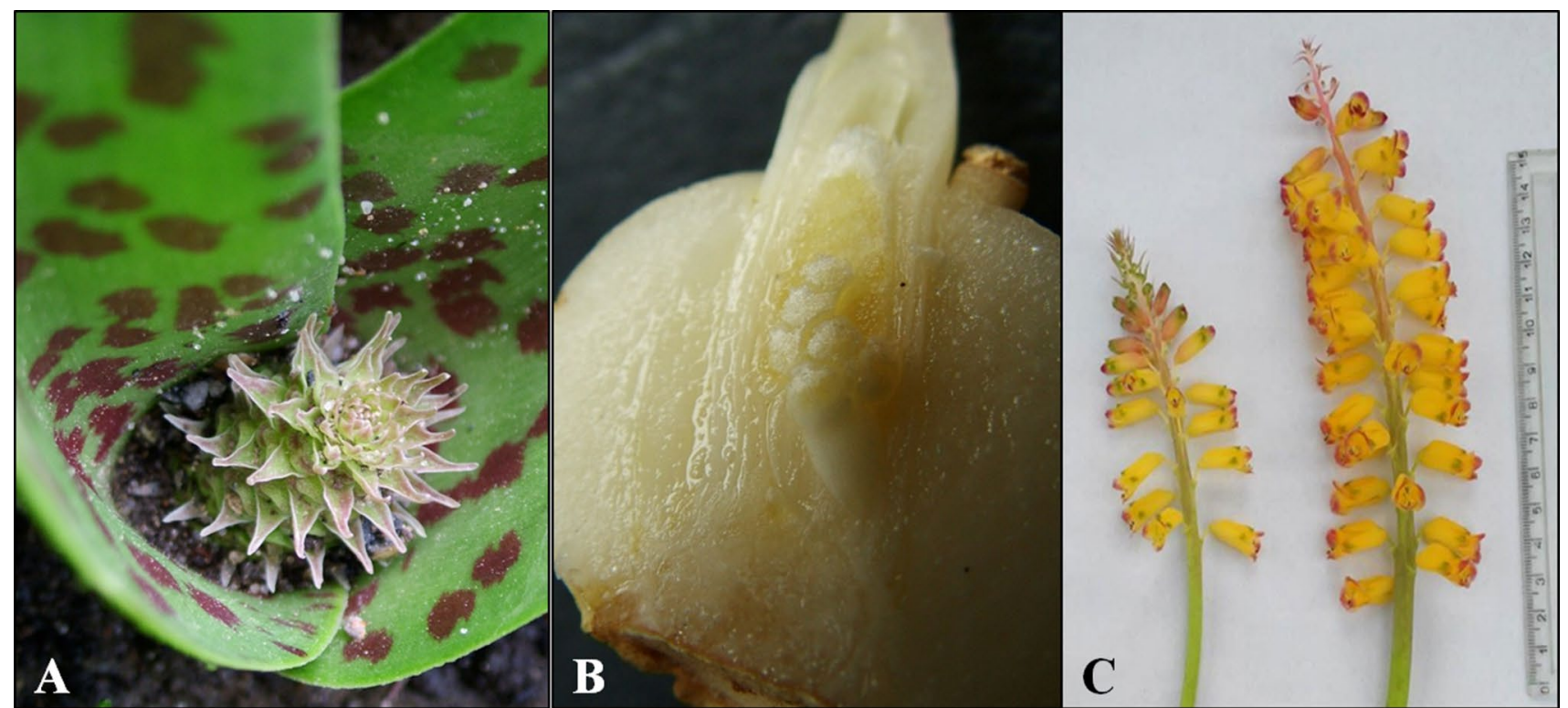

Fig. 3 a Stage with visible flower buds, $\mathbf{b}$ longitudinal section of a bulb with first flower primordia visible, $\mathbf{c}$ inflorescence of lachenalia 'Josephine' (left, control; right, irradiation)

at $105^{\circ} \mathrm{C}$ until constant weight was reached. Dry weights of bulbs, roots, and leaves were determined two 2 months after planting the bulbs, and the dry weight of inflorescence stems was assessed during plant flowering. Three analytical replications were performed for each treatment.

\subsection{Statistical analysis}

All data were analyzed using the STATISTICA 10.0 data analysis software system (StatSoft, Tulsa, OK, USA). Experimental data were subjected to analysis of variance, and Tukey's multiple range test was used to separate mean values at a significance level of $p \leq 0.05$. In addition, correlations between dry weight and plant pigment content were tested at a $p \leq 0.05$ using Pearson correlation coefficient.

\section{Results}

\subsection{Morphological parameters and time of flowering}

The first cultivars to produce visible flower buds (Fig. 3a), 47 days after bulb planting, were additionally irradiated 'Rainbow Bells', irradiated 'Josephine', and control. The period from bulb planting to first flower buds was the longest (61 days) in 'Cherise', irrespective of light conditions (Fig. 4). Under supplemental irradiation, 'Rainbow Bells', 'Aqua Lady', 'Josephine', and 'Riana' flowered earlier than the control plants by $19,18,7$, and 5 days, respectively. Supplemental irradiation did not accelerate flowering in
'Radient' and 'Cherise' cultivars, and they flowered at the latest only 94-98 days after planting.

An analysis of flowering time course revealed that, irrespective of light conditions, the bulbs of individual cultivars started to bloom at a similar time, usually covering 1 week. The flowering was most intense at the beginning of this period, when on average $50 \%$ of bulbs of each cultivar bloomed (Fig. 5). Depending on light conditions and cultivar, lachenalia bulbs flowered from February 20th to March 24th. Figure 3B shows a longitudinal section of a bulb with visible first flower primordia, which proves that inflorescence primordia were formed at the stage of bulb storage as a result, all planted bulbs flowered.

Irradiated bulbs of all investigated cultivars produced shorter inflorescences and inflorescence stems than control bulbs. This difference was cultivar dependent and equaled 2.4-6.6 cm and 1.1-3.4 cm, respectively (Table 1). There were considerable differences between cultivars when compared to the control bulbs. For example, the shortest inflorescence stems were produced by 'Josephine' plants, which were by $14-15 \mathrm{~cm}$ shorter than the longest ones in this experiment produced by 'Cherise', 'Aqua Lady', and 'Rainbow'. Despite that, 'Josephine' inflorescences produced the same number of florets as 'Cherise' and even more than 'Rainbow Bells'. Plants cultivated in the chamber with additional irradiance formed more florets than those grown under natural light conditions (Fig. 3c). The greatest difference in this parameter was observed in 'Cherise', in which the irradiated plants produced 10 more florets than the control. Cultivar comparison also demonstrated that, irrespective of light conditions, the lowest number of florets was observed 


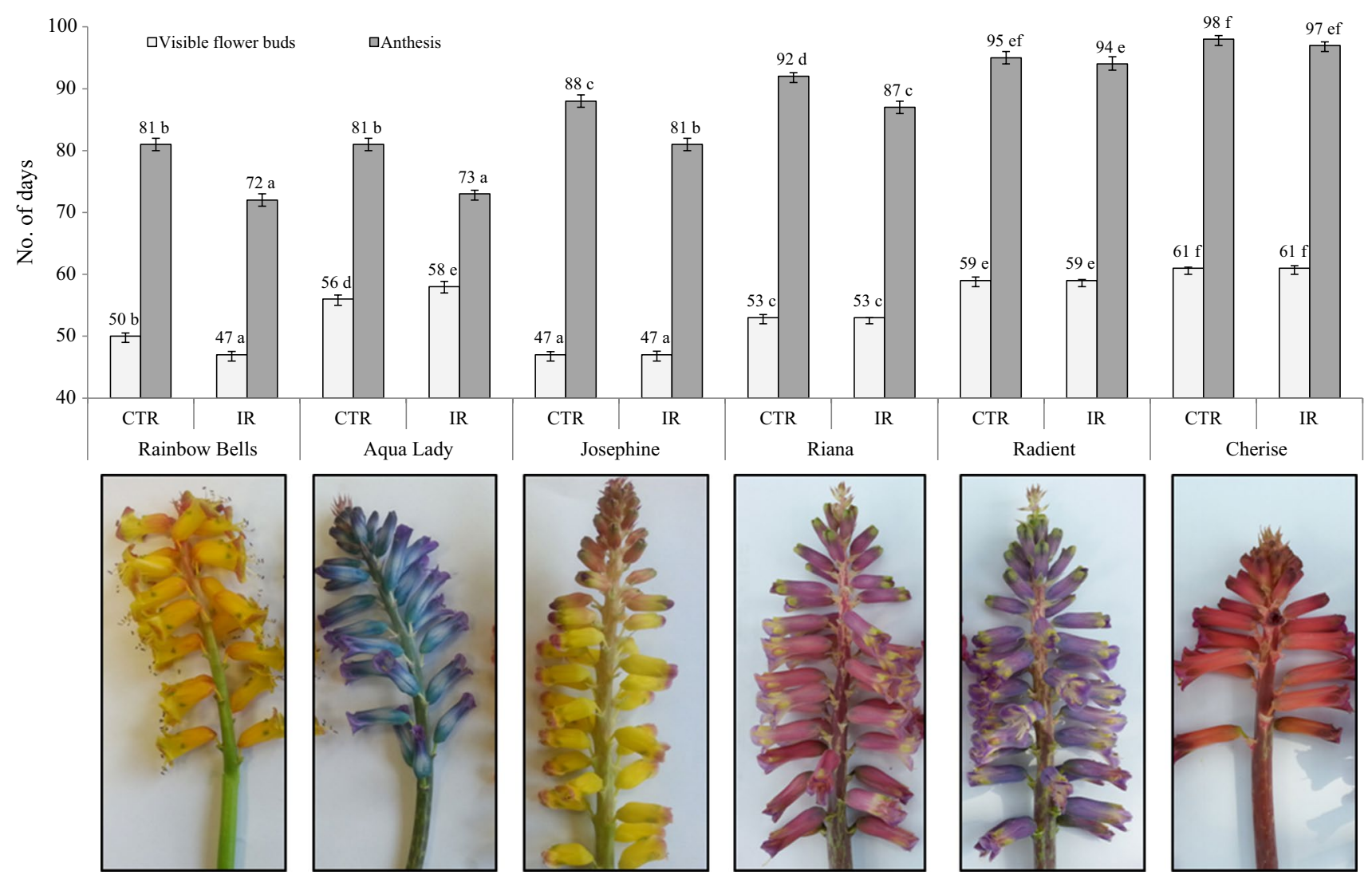

Fig. 4 Number of days to visible flower buds and anthesis of lachenalia grown under different light conditions. Treatment: control (CTR) or irradiation (IR). Bars represent standard deviation, and mean

in 'Rainbow Bells' (15.1-17.6) and the highest in 'Radient' (42.8-50.9). Four of the investigated cultivars ('Rainbow Bells', 'Josephine', 'Riana', and 'Cherise') positively responded to irradiation by forming longer florets than control plants. Moreover, irrespective of light conditions, the shortest florets were found in 'Josephine' and the longest in 'Cherise'. Visual quality reception of the inflorescences was analyzed by comparing the fullness ratio that was consistently better in the irradiated bulbs versus the control.

Irradiation positively affected thickness of the inflorescence stems, except for 'Rainbow Bells', which produced stems of the same thickness in both variants (Fig. 6). Among control plants, the thickest inflorescence stems were observed in 'Riana' and 'Radient' and the thinnest in 'Rainbow Bells', 'Aqua Lady', and 'Josephine'. Regardless of the cultivar and light conditions, first leaves emerged 5-7 days after planting (data not shown). Only in 'Rainbow Bells' did supplemental irradiation affect plant foliage by reducing the number of leaves produced by a single bulb (Table 2). The use of sodium lamps resulted in shortening of the first leaves (by $2-4 \mathrm{~cm}$ ) in 'Aqua Lady', 'Riana', 'Radient', and 'Cherise' and second leaves (by over $2 \mathrm{~cm}$ ) in 'Radient' and 'Cherise' compared to the control. The shortest leaves were values followed by a different letter(s) are significantly different at $p \leq 0.05$ according to Tukey's test

observed in 'Josephine', being on average two times shorter than in the other cultivars. The width of the first leaf was the same in all analyzed objects, but irradiation increased the width of the second leaf in all tested cultivars except for 'Aqua Lady'.

\subsection{Photosynthetic pigments}

Pigment content in the leaves of investigated lachenalia cultivars is presented in Table 3. The leaves of four cultivars, i.e. 'Aqua Lady', 'Riana', 'Radient', and 'Cherise', contained significantly more chlorophyll a than the control plants. The last cultivar also had higher chlorophyll $b$ content than its control counterpart. The use of the lamps increased the total amount of chlorophyll $\mathrm{a}$ and $\mathrm{b}$, as well as carotenoids in the leaves of 'Riana', 'Radient', and 'Cherise' plants.

\subsection{Dry weight}

An analysis of dry weight content in individual plant organs revealed that bulbs and leaves of all investigated cultivars grown under additional irradiation were characterized by greater values of this parameter compared to the control 


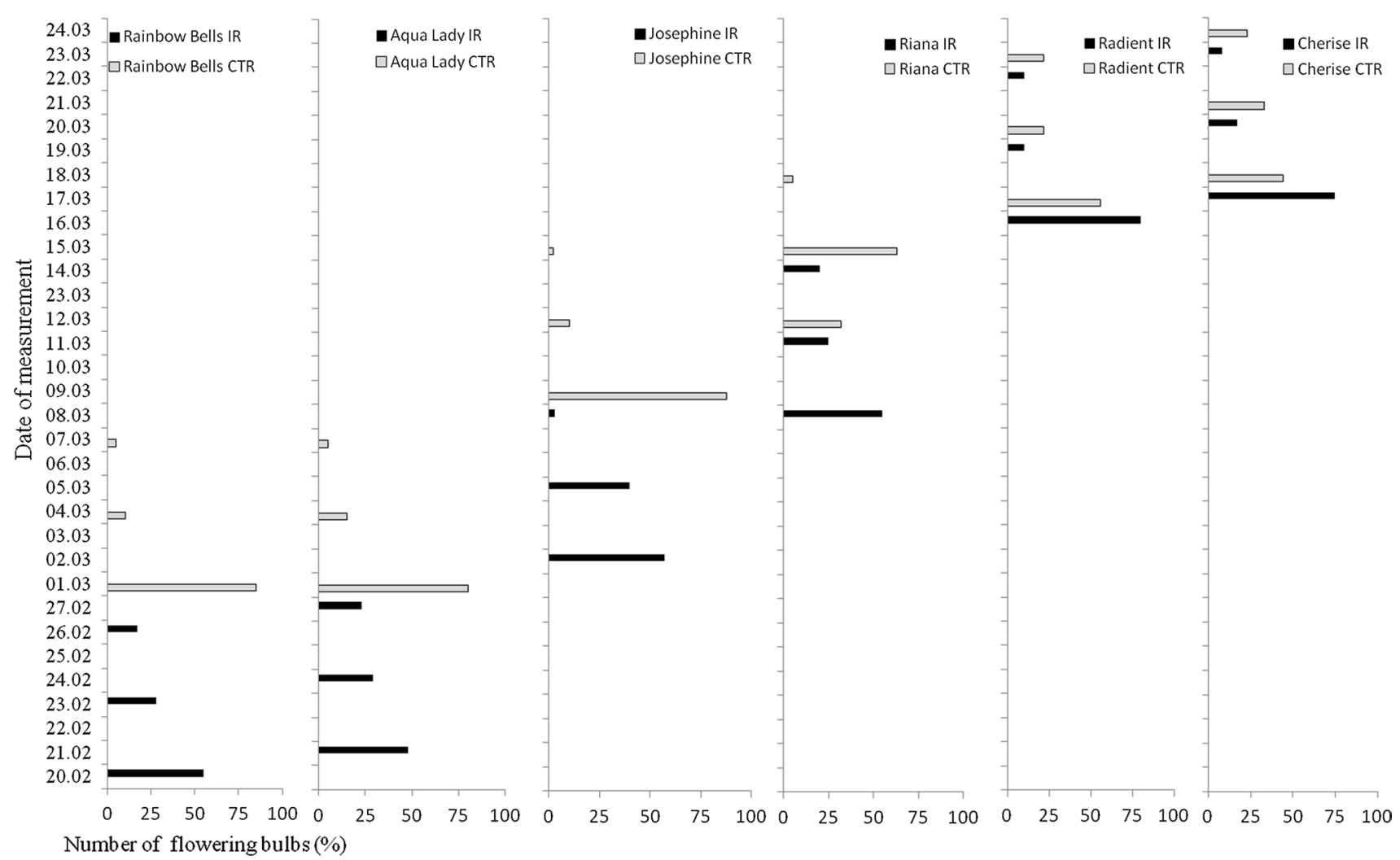

Fig. 5 Time course of flowering in lachenalia grown under different light conditions. Treatment: control (CTR) and irradiation (IR)

Table 1 Inflorescence quality of lachenalia grown under different light conditions

\begin{tabular}{|c|c|c|c|c|c|c|}
\hline Cultivar & Treatment & $\begin{array}{l}\text { Inflorescence stem } \\
\text { height }(\mathrm{cm})\end{array}$ & $\begin{array}{l}\text { Inflorescence } \\
\text { length }(\mathrm{cm})\end{array}$ & Florets (no.) & Floret length $(\mathrm{cm})$ & Fullness ratio \\
\hline \multirow[t]{2}{*}{ 'Rainbow Bells' } & $\mathrm{CTR}^{\mathrm{w}}$ & $39.6 \pm 0.4 \mathrm{f}^{\mathrm{y}}$ & $7.3 \pm 0.2 \mathrm{c}$ & $15.1 \pm 0.1 \mathrm{a}$ & $2.6 \pm 0.1 \mathrm{~d}$ & $2.1 \pm 0.1 \mathrm{a}$ \\
\hline & $\mathrm{IR}^{\mathrm{x}}$ & $37.0 \pm 0.4 \mathrm{e}$ & $5.8 \pm 0.1 \mathrm{a}$ & $17.6 \pm 0.5 \mathrm{~b}$ & $2.7 \pm 0.1 \mathrm{e}$ & $3.1 \pm 0.1 \mathrm{~d}$ \\
\hline \multirow[t]{2}{*}{ ‘Aqua Lady’ } & CTR & $39.4 \pm 0.5 \mathrm{f}$ & $9.4 \pm 0.2 \mathrm{e}$ & $24.0 \pm 0.8 \mathrm{~d}$ & $2.5 \pm 0.1 \mathrm{~cd}$ & $2.6 \pm 0.1 \mathrm{bc}$ \\
\hline & IR & $36.3 \pm 0.9 \mathrm{e}$ & $6.0 \pm 0.2 \mathrm{ab}$ & $29.2 \pm 0.5 \mathrm{f}$ & $2.6 \pm 0.1 \mathrm{~d}$ & $4.8 \pm 0.1 \mathrm{f}$ \\
\hline \multirow[t]{2}{*}{ 'Josephine' } & CTR & $24.6 \pm 0.3 \mathrm{~b}$ & $7.2 \pm 0.3 \mathrm{c}$ & $20.6 \pm 0.4 \mathrm{c}$ & $1.9 \pm 0.1 \mathrm{a}$ & $2.9 \pm 0.1 \mathrm{~cd}$ \\
\hline & IR & $22.1 \pm 0.8 \mathrm{a}$ & $6.6 \pm 0.4 b$ & $26.4 \pm 0.4 \mathrm{e}$ & $2.1 \pm 0.1 \mathrm{~b}$ & $4.1 \pm 0.2 \mathrm{e}$ \\
\hline \multirow[t]{2}{*}{ 'Riana' } & CTR & $30.7 \pm 0.5 \mathrm{~d}$ & $7.8 \pm 0.2 \mathrm{~cd}$ & $32.8 \pm 0.6 \mathrm{~g}$ & $2.5 \pm 0.1 \mathrm{~cd}$ & $4.2 \pm 0.2 \mathrm{e}$ \\
\hline & IR & $27.0 \pm 0.4 \mathrm{c}$ & $6.6 \pm 0.1 \mathrm{~b}$ & $37.5 \pm 0.2 \mathrm{~h}$ & $2.8 \pm 0.1 \mathrm{e}$ & $5.7 \pm 0.1 \mathrm{~h}$ \\
\hline \multirow[t]{2}{*}{ 'Radient' } & CTR & $26.4 \pm 0.5 \mathrm{c}$ & $9.4 \pm 0.5 \mathrm{e}$ & $42.8 \pm 0.6 \mathrm{i}$ & $2.4 \pm 0.1 \mathrm{c}$ & $4.6 \pm 0.2 \mathrm{f}$ \\
\hline & IR & $24.0 \pm 0.8 \mathrm{~b}$ & $8.3 \pm 0.3 \mathrm{~d}$ & $50.9 \pm 0.8 \mathrm{j}$ & $2.5 \pm 0.1 \mathrm{~cd}$ & $6.2 \pm 0.2 \mathrm{i}$ \\
\hline \multirow[t]{2}{*}{ 'Cherise' } & CTR & $38.6 \pm 0.4 \mathrm{f}$ & $9.0 \pm 0.1 \mathrm{e}$ & $21.7 \pm 0.5 \mathrm{c}$ & $2.9 \pm 0.1 \mathrm{f}$ & $2.3 \pm 0.1 \mathrm{ab}$ \\
\hline & IR & $32.0 \pm 0.8 \mathrm{~d}$ & $6.1 \pm 0.1 \mathrm{ab}$ & $31.5 \pm 0.4 \mathrm{~g}$ & $3.1 \pm 0.1 \mathrm{~g}$ & $5.2 \pm 0.1 \mathrm{~g}$ \\
\hline \multicolumn{7}{|l|}{ Main effects ${ }^{\mathrm{z}}$} \\
\hline Cultivar & & $<0.0001$ & $<0.0001$ & $<0.0001$ & $<0.0001$ & $<0.0001$ \\
\hline Treatment & & $<0.0001$ & $<0.0001$ & $<0.0001$ & $<0.0001$ & $<0.0001$ \\
\hline Cultivar $\times$ Treatment & & $<0.0001$ & $<0.0001$ & $<0.0001$ & $<0.0001$ & $<0.0001$ \\
\hline
\end{tabular}

${ }^{\mathrm{w}} \mathrm{CTR}$, control

${ }^{x}$ IR, irradiation

${ }^{\mathrm{y}}$ Mean values $( \pm \mathrm{SD})$ in columns followed by different letter(s) are significantly different at $p \leq 0.05$ according to Tukey's test

${ }^{\mathrm{z}}$ Significant effects $(p \leq 0.05)$ 


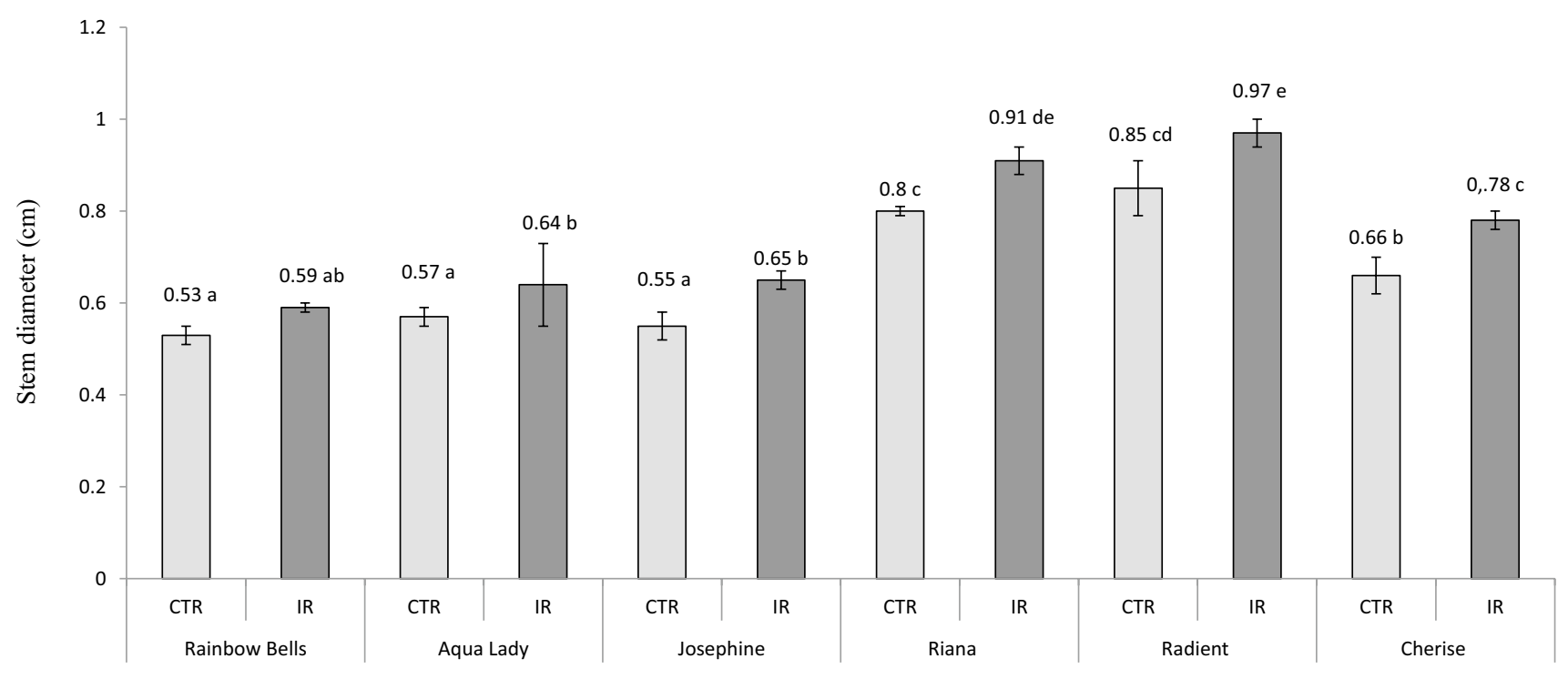

Fig. 6 Inflorescence stem diameter of lachenalia grown under different light conditions. Treatment: control (CTR) and irradiation (IR). Bars represent standard deviation, and mean values followed by a different letter(s) are significantly different at $p \leq 0.05$ according to Tukey's test

Table 2 Number of leaves and leaf quality in lachenalia grown under different light conditions

\begin{tabular}{|c|c|c|c|c|c|c|}
\hline Cultivar & Treatment & Leaf (no.) & First leaf length $(\mathrm{cm})$ & First leaf width $(\mathrm{cm})$ & Second leaf length $(\mathrm{cm})$ & Second leaf width $(\mathrm{cm})$ \\
\hline \multirow[t]{2}{*}{ 'Rainbow Bells' } & $\mathrm{CTR}^{\mathrm{w}}$ & $2.4 \pm 0.4 b^{y}$ & $29.5 \pm 1.3 \mathrm{de}$ & $3.5 \pm 0.1 \mathrm{a}$ & $32.8 \pm 0.5$ ef & $2.4 \pm 0.1 \mathrm{c}$ \\
\hline & $\mathrm{IR}^{\mathrm{x}}$ & $2.0 \pm 01 \mathrm{a}$ & $29.4 \pm 1.1 \mathrm{de}$ & $3.5 \pm 0.1 \mathrm{a}$ & $32.7 \pm 0.8$ ef & $2.5 \pm 0.1 \mathrm{~d}$ \\
\hline \multirow[t]{2}{*}{ 'Aqua Lady' } & CTR & $2.5 \pm 0.1 \mathrm{~b}$ & $30.2 \pm 0.5$ ef & $3.7 \pm 0.1 \mathrm{a}$ & $32.9 \pm 0.5 \mathrm{f}$ & $2.5 \pm 0.1 \mathrm{de}$ \\
\hline & IR & $2.2 \pm 0.1 \mathrm{ab}$ & $28.2 \pm 0.5 \mathrm{~d}$ & $3.9 \pm 0.1 \mathrm{a}$ & $32.7 \pm 0.8 \mathrm{ef}$ & $2.5 \pm 0.1 \mathrm{de}$ \\
\hline \multirow[t]{2}{*}{ 'Josephine' } & CTR & $2.5 \pm 0.1 \mathrm{~b}$ & $16.7 \pm 0.2 \mathrm{a}$ & $3.3 \pm 0.1 \mathrm{a}$ & $17.7 \pm 0.4 \mathrm{a}$ & $2.1 \pm 0.1 \mathrm{a}$ \\
\hline & IR & $2.5 \pm 0.1 \mathrm{~b}$ & $16.6 \pm 0.2 \mathrm{a}$ & $3.6 \pm 0.1 \mathrm{a}$ & $17.8 \pm 0.1 \mathrm{a}$ & $2.3 \pm 0.1 \mathrm{~b}$ \\
\hline \multirow[t]{2}{*}{ 'Riana' } & CTR & $2.0 \pm 0.1 \mathrm{a}$ & $31.5 \pm 0.6 \mathrm{fg}$ & $3.8 \pm 0.1 \mathrm{a}$ & $31.4 \pm 0.5 \mathrm{de}$ & $2.7 \pm 0.1 \mathrm{f}$ \\
\hline & IR & $2.1 \pm 0.1 \mathrm{a}$ & $29.6 \pm 0.5 \mathrm{de}$ & $3.8 \pm 0.1 \mathrm{a}$ & $30.2 \pm 0.9 \mathrm{~d}$ & $2.9 \pm 0.1 \mathrm{gh}$ \\
\hline \multirow[t]{2}{*}{ 'Radient' } & CTR & $2.5 \pm 0.1 \mathrm{~b}$ & $25.6 \pm 0.5 \mathrm{c}$ & $3.8 \pm 0.1 \mathrm{a}$ & $26.8 \pm 0.3 \mathrm{c}$ & $2.7 \pm 0.1 \mathrm{fg}$ \\
\hline & IR & $2.3 \pm 0.1 \mathrm{ab}$ & $22.6 \pm 0.8 \mathrm{~b}$ & $3.6 \pm 0.1 \mathrm{a}$ & $24.4 \pm 0.9 \mathrm{~b}$ & $2.9 \pm 0.1 \mathrm{~h}$ \\
\hline \multirow[t]{2}{*}{ 'Cherise' } & CTR & $2.1 \pm 0.1 \mathrm{a}$ & $32.4 \pm 0.4 \mathrm{~g}$ & $3.8 \pm 0.1 \mathrm{a}$ & $34.6 \pm 0.5 \mathrm{~g}$ & $2.2 \pm 0.1 \mathrm{ab}$ \\
\hline & IR & $2.1 \pm 0.1 \mathrm{a}$ & $28.3 \pm 0.4 \mathrm{~d}$ & $4.0 \pm 0.1 \mathrm{a}$ & $32.2 \pm 0.3 \mathrm{ef}$ & $2.6 \pm 0.1 \mathrm{ef}$ \\
\hline \multicolumn{7}{|l|}{ Main effects ${ }^{\mathrm{z}}$} \\
\hline \multirow{3}{*}{$\begin{array}{l}\text { Cultivar } \\
\text { Treatment } \\
\text { Cultivar } \times \text { Treatment }\end{array}$} & & $<0.0001$ & $<0.0001$ & 0.037 & $<0.0001$ & $<0.0001$ \\
\hline & & 0.0002 & $<0.0001$ & NS & $<0.0001$ & $<0.0001$ \\
\hline & & 0.0048 & $<0.0001$ & NS & $<0.0001$ & $<0.0001$ \\
\hline
\end{tabular}

${ }^{\mathrm{w}} \mathrm{CTR}$, control

${ }^{\mathrm{x}} \mathrm{IR}$, irradiation

${ }^{\mathrm{y}}$ Mean values ( $\pm \mathrm{SD}$ ) in columns followed by different letter(s) are significantly different at $p \leq 0.05$ according to Tukey's test

${ }^{\mathrm{z}}$ Significant effects $(p \leq 0.05)$; NS, not significant

(Table 4). The differences were particularly visible for bulbs, as they often reached a dozen or so percentage points ('Cherise', 'Radient', and 'Riana') in favor of the irradiated plants. No significant differences in dry weight of stems produced by irradiated and control plants were observed for 'Radient' and 'Cherise'. Additional irradiation caused an increase in dry weight of roots only in 'Riana'. A comparison of individual plant organs, irrespective of light conditions and cultivar, demonstrated that lachenalia bulbs had the greatest dry weight, followed by roots, and leaves and stems (Fig. 7).

Positive correlations were found between dry weight of bulbs and leaves and chlorophyll a and carotenoid content in the leaves. Other compounds were statistically insignificant (Table 5). 
Table 3 Plant pigment content in lachenalia leaves grown under different light conditions

\begin{tabular}{|c|c|c|c|c|c|}
\hline \multirow[t]{2}{*}{ Cultivar } & \multirow[t]{2}{*}{ Treatment } & \multicolumn{4}{|c|}{ Plant pigment content ( $\mathrm{mg} \mathrm{g}^{-1}$ fresh weight) } \\
\hline & & Chl a & Chl b & $\mathrm{Chl} \mathrm{a}+\mathrm{b}$ & Car \\
\hline \multirow[t]{2}{*}{ ‘Rainbow Bells’ } & $\mathrm{CTR}^{\mathrm{w}}$ & $0.437 \pm 0.029 b c^{y}$ & $0.178 \pm 0.012 b-d$ & $0.614 \pm 0.042 \mathrm{c}-\mathrm{e}$ & $0.113 \pm 0.003 \mathrm{~b}-\mathrm{d}$ \\
\hline & $\mathrm{IR}^{\mathrm{x}}$ & $0.508 \pm 0.008 \mathrm{~cd}$ & $0.204 \pm 0.004 \mathrm{c}-\mathrm{d}$ & $0.712 \pm 0.012 \mathrm{de}$ & $0.125 \pm 0.024 \mathrm{de}$ \\
\hline \multirow[t]{2}{*}{ 'Aqua Lady’ } & CTR & $0.444 \pm 0.003 \mathrm{bc}$ & $0.162 \pm 0.003 \mathrm{a}-\mathrm{c}$ & $0.606 \pm 0.006 \mathrm{~b}-\mathrm{e}$ & $0.104 \pm 0.001 \mathrm{~b}-\mathrm{d}$ \\
\hline & IR & $0.535 \pm 0.023 \mathrm{de}$ & $0.199 \pm 0.003 \mathrm{~cd}$ & $0.734 \pm 0.026$ ef & $0.124 \pm 0.008 \mathrm{~d}-\mathrm{e}$ \\
\hline \multirow[t]{2}{*}{ 'Josephine' } & CTR & $0.362 \pm 0.020 \mathrm{ab}$ & $0.129 \pm 0.064 \mathrm{ab}$ & $0.491 \pm 0.026 \mathrm{a}-\mathrm{c}$ & $0.086 \pm 0.005 \mathrm{ab}$ \\
\hline & IR & $0.354 \pm 0.037 \mathrm{ab}$ & $0.133 \pm 0.009 \mathrm{ab}$ & $0.488 \pm 0.045 \mathrm{a}-\mathrm{c}$ & $0.090 \pm 0.010 \mathrm{a}-\mathrm{c}$ \\
\hline \multirow[t]{2}{*}{ 'Riana' } & CTR & $0.338 \pm 0.017 \mathrm{a}$ & $0.116 \pm 0.004 \mathrm{a}$ & $0.453 \pm 0.019 \mathrm{a}$ & $0.074 \pm 0.004 \mathrm{a}$ \\
\hline & IR & $0.467 \pm 0.005 \mathrm{c}$ & $0.164 \pm 0.002 \mathrm{a}-\mathrm{c}$ & $0.632 \pm 0.005 \mathrm{de}$ & $0.114 \pm 0.001 \mathrm{~cd}$ \\
\hline \multirow[t]{2}{*}{ 'Radient' } & CTR & $0.323 \pm 0.014 \mathrm{a}$ & $0.143 \pm 0.041 \mathrm{ab}$ & $0.473 \pm 0.054 \mathrm{ab}$ & $0.068 \pm 0.011 \mathrm{a}$ \\
\hline & IR & $0.468 \pm 0.008 \mathrm{c}$ & $0.163 \pm 0.007 \mathrm{a}-\mathrm{c}$ & $0.631 \pm 0.001 \mathrm{de}$ & $0.109 \pm 0.003 \mathrm{bd}$ \\
\hline \multirow[t]{2}{*}{ 'Cherise' } & CTR & $0.417 \pm 0.047 \mathrm{bc}$ & $0.167 \pm 0.022 \mathrm{a}-\mathrm{c}$ & $0.584 \pm 0.068 \mathrm{a}-\mathrm{d}$ & $0.094 \pm 0.013 \mathrm{a}-\mathrm{c}$ \\
\hline & IR & $0.629 \pm 0.080 \mathrm{e}$ & $0.228 \pm 0.034 \mathrm{~d}$ & $0.857 \pm 0.114 \mathrm{f}$ & $0.151 \pm 0.019 \mathrm{e}$ \\
\hline \multicolumn{6}{|l|}{ Main effects ${ }^{z}$} \\
\hline Cultivar & & $<0.0001$ & $<0.0001$ & $<0.0001$ & $<0.0001$ \\
\hline Treatment & & $<0.0001$ & $<0.0001$ & $<0.0001$ & $<0.0001$ \\
\hline Cultivar $\times$ Treatment & & 0.0002 & NS & $<0.0001$ & 0.0004 \\
\hline
\end{tabular}

${ }^{\mathrm{w}} \mathrm{CTR}$, control

${ }^{\mathrm{x}} \mathrm{IR}$, irradiation

${ }^{\mathrm{y}}$ Mean values $( \pm \mathrm{SD})$ in columns followed by different letter(s) are significantly different according to Tukey's least significant difference test at $p \leq 0.05$

${ }^{\mathrm{z}}$ Significant effects $(p \leq 0.05)$; NS, not significant

Table 4 Dry weight of lachenalia organs grown under different light conditions

\begin{tabular}{|c|c|c|c|c|c|}
\hline \multirow[t]{2}{*}{ Cultivar } & \multirow[t]{2}{*}{ Treatment } & \multicolumn{4}{|c|}{ Dry weight (\%) } \\
\hline & & Bulb & Root & Leaf & Stem \\
\hline \multirow[t]{2}{*}{ 'Rainbow Bells' } & $\mathrm{CTR}^{\mathrm{w}}$ & $14.4 \pm 0.5 \mathrm{ab}^{\mathrm{y}}$ & $7.3 \pm 0.1 \mathrm{a}-\mathrm{d}$ & $3.2 \pm 0.2 \mathrm{ab}$ & $4.4 \pm 0.3 \mathrm{a}-\mathrm{c}$ \\
\hline & $\mathrm{IR}^{\mathrm{x}}$ & $20.9 \pm 2.9 \mathrm{c}$ & $7.8 \pm 0.2 b-d$ & $4.4 \pm 0.3 \mathrm{~cd}$ & $6.5 \pm 0.4 \mathrm{~d}$ \\
\hline \multirow[t]{2}{*}{ 'Aqua Lady' } & CTR & $12.4 \pm 2.7 \mathrm{a}$ & $5.7 \pm 0.6 \mathrm{ab}$ & $3.2 \pm 0.2 \mathrm{ab}$ & $3.5 \pm 0.4 \mathrm{a}$ \\
\hline & IR & $19.6 \pm 1.9 \mathrm{bc}$ & $7.6 \pm 0.5 \mathrm{a}-\mathrm{d}$ & $4.7 \pm 0.7 \mathrm{~d}$ & $5.5 \pm 0.2 \mathrm{~cd}$ \\
\hline \multirow[t]{2}{*}{ 'Josephine' } & CTR & $13.3 \pm 2.1 \mathrm{ab}$ & $6.6 \pm 0.5 \mathrm{a}-\mathrm{c}$ & $3.7 \pm 0.2 b c$ & $5.1 \pm 1.2 \mathrm{c}$ \\
\hline & IR & $23.8 \pm 2.0 \mathrm{c}$ & $7.0 \pm 0.2 \mathrm{a}-\mathrm{d}$ & $4.8 \pm 0.2 \mathrm{~d}$ & $6.7 \pm 0.1 \mathrm{~d}$ \\
\hline \multirow[t]{2}{*}{ 'Riana' } & CTR & $11.1 \pm 2.0 \mathrm{a}$ & $6.4 \pm 1.5 \mathrm{a}-\mathrm{c}$ & $3.0 \pm 0.2 \mathrm{ab}$ & $3.7 \pm 0.3 \mathrm{ab}$ \\
\hline & IR & $22.5 \pm 4.8 \mathrm{c}$ & $9.1 \pm 1.7 \mathrm{~d}$ & $4.1 \pm 0.4 \mathrm{~cd}$ & $4.9 \pm 0.3 \mathrm{c}$ \\
\hline \multirow[t]{2}{*}{ 'Radient' } & CTR & $10.8 \pm 2.9 \mathrm{a}$ & $6.7 \pm 1.5 \mathrm{a}-\mathrm{d}$ & $2.8 \pm 0.1 \mathrm{a}$ & $5.0 \pm 0.3 \mathrm{c}$ \\
\hline & IR & $22.1 \pm 1.4 \mathrm{c}$ & $8.3 \pm 1.7 \mathrm{c}-\mathrm{d}$ & $4.2 \pm 0.1 \mathrm{~cd}$ & $5.2 \pm 0.1 \mathrm{c}$ \\
\hline \multirow[t]{2}{*}{ 'Cherise' } & CTR & $7.9 \pm 0.9 \mathrm{a}$ & $5.2 \pm 1.5 \mathrm{a}$ & $2.7 \pm 0.3 \mathrm{a}$ & $4.3 \pm 0.2 \mathrm{a}-\mathrm{c}$ \\
\hline & IR & $21.4 \pm 1.0 \mathrm{c}$ & $7.0 \pm 0.2 \mathrm{a}-\mathrm{d}$ & $4.3 \pm 0.3 \mathrm{~cd}$ & $5.4 \pm 0.1 \mathrm{~cd}$ \\
\hline \multicolumn{6}{|l|}{ Main effects ${ }^{z}$} \\
\hline Cultivar & & NS & 0.0177 & 0.0267 & $<0.0001$ \\
\hline Treatment & & $<0.0001$ & $<0.0001$ & $<0.0001$ & $<0.0001$ \\
\hline Cultivar $\times$ Treatment & & NS & NS & NS & 0.0087 \\
\hline
\end{tabular}

${ }^{\mathrm{w}} \mathrm{CTR}$, control

${ }^{\mathrm{x}} \mathrm{IR}$, irradiation

${ }^{y}$ Mean values $( \pm S D)$ in columns followed by different letter(s) are significantly different according to Tukey's least significant difference test at $p \leq 0.05$

${ }^{\mathrm{z}}$ Significant effects $(p \leq 0.05)$; NS, not significant 


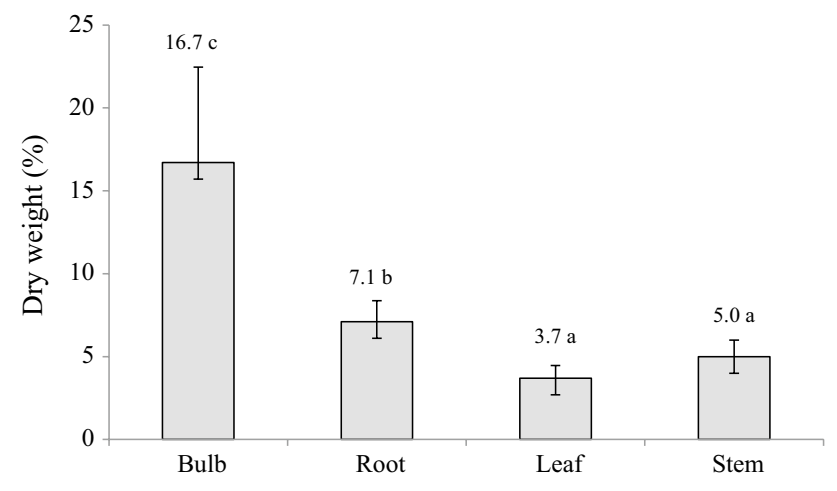

Fig. 7 Dry weight of lachenalia organs irrespective of light conditions and cultivar (sample size $2.0 \mathrm{~g}$ ). Bars represent standard deviation, and mean values followed by a different letter are significantly different at $p \leq 0.05$ according to Tukey's test

Table 5 Correlation coefficients between dry weight content (\%) and plant pigment content $\left(\mathrm{mg} \mathrm{g}^{-1}\right.$ fresh weight), irrespective of the cultivar and light conditions

\begin{tabular}{lllll}
\hline Dry weight & Chl a & Chl b & Chl a $+\mathrm{b}$ & Car \\
\hline Bulb & $0.384^{\mathrm{z}}$ & 0.304 & 0.326 & $0.370^{\mathrm{z}}$ \\
Root & 0.179 & 0.037 & 0.128 & 0.236 \\
Leaf & $0.378^{\mathrm{z}}$ & 0.196 & 0.276 & $0.392^{\mathrm{z}}$ \\
Stem & 0.172 & 0.102 & 0.057 & 0.143 \\
\hline
\end{tabular}

${ }^{\mathrm{z}}$ Correlations significant at $p \leq 0.05$

\section{Discussion}

\subsection{Morphological parameters and flowering time}

Greenhouse cultivation offers flexibility and enables crops to be grown at diverse geographical latitudes. However, exposing the crops to light climates they have not experienced in their natural environment may result in individual genotypes responding differently to growing conditions (van Ieperen 2016), as confirmed by previous reports on lachenalia cultivation (Kapczyńska 2012, 2014). In this study, irrespective of light treatment and cultivar, all planted lachenalia bulbs developed foliage and inflorescence stems, which could be due to the fact that temperature range applied during the bulb dormancy period was conducive to organogenesis. Our results showed that light environment modulated inflorescence stem quality and flower development. The investigated lachenalia cultivars began flowering from the second half of February to the second half of March, but in four of the investigated cultivars, the use of sodium lamps accelerated flowering by a few to even a dozen or so days (early flowering shortens the production process, enabling the start of the next cultivation cycle). Previous studies also indicated that reduced irradiance delayed the flowering time in Eustoma (Islam et al. 2005), Phalaenopsis (Wu et al. 2013), Calendula, Phlox, Papaver (Baloch et al. 2014), and Brodiaea (Han et al. 1991). The delay of inflorescence stem emergence in low irradiance conditions may be the result of decreased availability of sucrose $(\mathrm{Wu}$ et al. 2013). A strong red/far-red region of conventional lamps is utilized by plants to control photomorphogenetic responses through mediation of phytochromes (Sowmya et al. 2017). The blue light receptor gene (cryptochrome 2), which promotes flowering of Arabidopsis, requires redlight signaling to promote floral advancement (Izawa et al. 2002). Hidaka et al. (2015) reported that the period from flower bud differentiation to anthesis of Fragaria $\times$ ananassa plants was shortened by supplemental lighting, which caused an acceleration of leaf photosynthesis and promoted rapid plant growth; as a result, a tendency for early flowering was observed. In our study, a comparison of additionally irradiated plants identified early and late flowering lachenalia cultivars. 'Rainbow Bells' and 'Aqua Lady' plants flowered markedly earlier (on average after 72-73 days) than 'Josephine' and 'Riana' (on average after 83-87 days), and 'Radient' and 'Cherise' were the last to bloom (on average after 94-97 days). Variability in flowering time in other lachenalia cultivars was reported by Kapczyńska (2012, 2013, 2014), Kapczynska and Malik (2016), Kapczynska and Kidawska (2016), and Ruffoni et al. (2013). By planting bulbs of different lachenalia cultivars on the same time, the flowering spread over time may be obtained. Based on our results, supplemental irradiation is recommended at this time of year for better quality potted plants, as the irradiated lachenalias had shorter and thicker inflorescence stems with more individual florets in the inflorescence. Dueck et al. (2016) reported that light treatment (HPS lamps) with a relatively high red-tofar-red ratio and, hence, high phytochrome photostationary state value in Phalaenopsis, abolished (by modulating auxin transport via phytochrome B) apical dominance of the primary flowering inflorescence, allowing for more inflorescences to develop. Similar mechanisms might have occurred in irradiated lachenalia plants that developed more florets on inflorescence stems. Moreover, high light intensity promoted vegetative plant development; consequently, an abundant flowering response was observed (Islam et al. 2005). In this study, all cultivars responded to the supplemental irradiation by increasing the fullness ratio of the inflorescence stem, which considerably affected the decorative value of the plants. Our results also demonstrated that the differences in the number of florets or fullness ratio were due to genetic differences between cultivars. 
Roh et al. (1995) adopted plant compactness, understood as short floral stems and leaves at the flowering stage, as a determinant of lachenalia quality. In this study, supplemental irradiation resulted in shortening the first leaves by $7-13 \%$ in four investigated cultivars. A distinctive cultivar in terms of leaf length was 'Josephine', in which the leaves were nearly half as short as in the other cultivars, irrespective of light conditions. Roh (2005) claimed that the leaf length in lachenalia may also differ depending on storage and growing temperature; for example, the leaves of lachenalia 'Pearsonii' were longer as forcing temperatures increased.

\subsection{Photosynthetic pigments}

Our experiment revealed that tested cultivars reacted to supplemental irradiation by increasing the level of photosynthetic pigments compared to control plants, but the differences were significant in only a few cases. The leaves of four cultivars had a higher chlorophyll a content, one cultivar was richer in chlorophyll $b$, and three cultivars had a higher carotenoid content. The content of all three photosynthetic pigments was enhanced by irradiation in 'Cherise'. The lamps provided mainly yellow (570-590 nm), orange (590-630 nm), and red (630-750 nm) light. Śmigielska et al. (2014) proved that under yellow light the contents of chlorophyll $\mathrm{a}, \mathrm{b}$, and $\mathrm{a}+\mathrm{b}$ were higher than under white or blue light in the leaves of Hyacinthus orientalis 'Anna Marie', 'Blue Star', and 'White Perl'. They also found a high chlorophyll content in leaf dry weight collected from plants of 'Blue Star' and 'White Perl' cultivated under red light. In addition, Shuai et al. (2016) reported that chlorophyll content in Vitis vinifera leaves increased in red light compared to the control (no supplemental lighting) and that the degradation of chloroplast ultrastructure was delayed in leaves grown under red light compared to blue light and control treatment. Leong et al. (1985) noticed a higher chlorophyll a/b ratio in Asplenium australasicum fronds under red light than those grown in blue light. These authors showed that the higher chlorophyll $\mathrm{a} / \mathrm{b}$ ratio in plants grown in red light was accompanied inter alia by higher whole-chain electron transport rates and higher photosynthetic rates in intact fern fronds.

\subsection{Dry weight}

Our study demonstrated a positive effect of irradiation on the dry weight of lachenalia leaves and bulbs. This may be explained by a positive physiological response of plants to a more favorable share of light and dark periods in the circadian rhythm. Adams and Langton (2005) identified possible mechanisms that increase plant dry weight such as long-day growth, which influences assimilate partitioning, and increased chlorophyll content per unit leaf area, which enhances photosynthesis. These mechanisms were also noticed in our study, as positive correlations between dry weight of bulbs and leaves and leaf content of chlorophyll a and carotenoids were observed.

In summary, lachenalia is an ornamental bulbous plant with substantial agronomic potential due to its extremely wide color palette and inflorescence durability. In horticulture, the final quality of plants and timing of flowering are the most important criteria by which optimization of environmental factors is conducted. To improve the quality of potted lachenalia and to control the flowering time, growers should consider using supplemental irradiation when cultivating lachenalia during months with little light. Our results indicated that providing better light conditions from HPS lamps positively affected plant compactness, inflorescence quality, plant dry weight, and photosynthetic pigment content when solar light was limited. The results also demonstrated that the variation in the observed features was due to genetic differences between the investigated cultivars.

Acknowledgements This work was supported by Polish Ministry of Science and Higher Education in Poland (DS 3500/KRO/2012-2015).

Open Access This article is distributed under the terms of the Creative Commons Attribution 4.0 International License (http://creativeco mmons.org/licenses/by/4.0/), which permits unrestricted use, distribution, and reproduction in any medium, provided you give appropriate credit to the original author(s) and the source, provide a link to the Creative Commons license, and indicate if changes were made.

\section{References}

Adams SR, Langton FA (2005) Photoperiod and plant growth: a review. J Hortic Sci Biotechnol 80:2-10

Arnon DT (1949) Copper enzyme in isolated chloroplasts polyphenoloxidase in Beta vulgaris L. Plant Physiol 24:1-15

Baloch J, Munir M, Farzana B (2014) Effects of supplementary irradiance on flowering time of obligate day ornamental annuals under non-inductive environment. Pak J Bot 46:1253-1259

Bergstrand KJ, Mortensen LM, Suthaparan A, Gislerød HR (2016) Acclimatisation of greenhouse crops to differing light quality. Sci Hortic 204:1-7

Catley JL, Brooking IR, Davies EA, Halligan EA (2002) Temperature and light requirements for Sandersonia aurantiaca flowering. Acta Hortic 570:105-112

Dueck T, Trouwborst G, Hogewoning SW, Meinen E (2016) Can a high red: far red ratio replace temperature-induced inflorescence development in Phalaenopsis? Environ Exp Bot 121:139-144

Duncan GD (2012) Botanical magazine monograph: the genus Lachenalia. Kew Publishing, Royal Botanic Gardens, Kew, p 479

Duncan GD (2013) Geophyte research and prouction in South Africa. In: Kamenetsky R, Okubo H (eds) Ornamental geophytes. From basic science to sustainable production. CRC Press, Boca Raton, pp 233-259

Grace OM, Van Staden J (2003) A horticultural history of Lachenalia (Hyacinthaceae). S Afr J Sci 99:526-531

Halevy AH (1990) Recent advances in control of flowering and growth habit of geophytes. Acta Hortic 266:35-42 
Han SS, Halevy AH, Sachs RM, Reid MS (1991) Flowering and corm yield of Brodiaea in response to temperature, photoperiod, corm size and planting depth. J Am Soc Hort Sci 116:19-22

Heuvelink E, Bakker MJ, Hogendonk L, Janse J, Kaarsemaker R, Maaswinkel R (2006) Horticultural lighting in the Netherlands: new developments. Acta Hortic 711:25-34

Hidaka K, Dan K, Imamura H, Takayama T, Sameshima K, Okimura M (2015) Variety comparison of effect of supplemental lighting with LED on growth and yield in forcing culture of strawberry. Environ Control Biol 53:135-143

Islam N, Patil G, Gislerød HR (2005) Effect of photoperiod and light integral on flowering and growth of Eustoma grandiflorum (Raf.) Shinn. Sci Hortic 103:441-451

Izawa T, Oikawa T, Sugiyama N, Tanisaka T, Yano M, Shimamoto K (2002) Phytochrome mediates the external light signal to repress $F T$ orthologs in photoperiodic flowering of rice. Genes Dev 16:2006-2020

Kapczyńska A (2012) Effect of planting time on flowering of four Lachenalia cultivars. Acta Hortic 937:575-579

Kapczyńska A (2013) Effect of plant spacing on the growth, flowering and bulb production of four lachenalia cultivars. S Afr J Bot 88:164-169

Kapczyńska A (2014) Effect of planting term on growth and flowering of two cultivars of lachenalia produced in a greenhouse as potting plants during winter months. J Hortic Res 22:29-34

Kapczyńska A, Kidawska A (2016) 'Namakwa' lachenalia's response to flurprimidol and different planting dates. Folia Hortic 28:173-179

Kapczyńska A, Malik M (2016) Planting time and flurprimidol treatment influence the growth and flowering of Lachenalia. HortTechnology 26:293-298

Kleynhans R (2006) Lachenalia spp. In: Anderson NO (ed) Flower breeding \& genetics: issues, challenges, and opportunities for the 21 st century. Springer, Dordrecht, pp 491-516

Kleynhans R, Niederwieser JG, Hancke FL (2002) Lachenalia: development and commercialization of a new flower bulb crop. Acta Hortic 570:81-85

Kleynhans R, Spies P, Spies JJ (2012) Cytogenetic and phylogenetic review of the genus Lachenalia. Floricult Ornam Biotechnol 6:98-115

Leong TY, Goodchild DJ, Anderson JM (1985) Effect of light quality on the composition, function, and structure of photosynthetic thylakoid membranes of Asplenium australasicum (Sm.) Hook. Plant Physiol 78:561-567
Lopéz J, Gonzales A, Fernandez JA, Franco JA, Banon S (2006) Effect of day length and corm storage temperature on flowering Gladiolus tristis sbsp. concolor. Acta Hortic 711:241-246

Roh MS (2005) Flowering and inflorescence development of Lachenalia aloides 'Pearsonii' as influenced by bulb storage and forcing temperature. Sci Hortic 104:305-323

Roh MS, Lawson RH, Song CY, Louw E (1995) Forcing Lachenalia as potted plant. Acta Hortic 397:147-153

Ruffoni B, Cervelli C, Kleynhans R, Fancke FL (2013) Forcing trials of Lachenalia cultivars in mediterranean area. Acta Hortic 1000:277-283

Schwend T, Prucker D, Mempel H (2015) Red light promotes compact growth of sunflowers. Eur J Hortic Sci 80:56-61

Shuai W, Xiaodi W, Xiangbin S, Baoliang W, Xiaocui Z, Haibo W, Fengzhi L (2016) Red and blue lights significantly affect photosynthetic properties and ultrastructure of mesophyll cells in senescing grape leaves. Hortic Plant J 2:82-90

Śmigielska M, Jerzy M, Krzymińska A (2014) The growth and flowering of Hyacinthus orientalis L. forced in pots under fluorescent light of different colours. Acta Agrobot 67:75-82

Sowmya R, Kurian Ciji Pearl, Narasimhan S, Srividya R (2017) Effect of light parameters on plant growth-a review. Jt Int Conf (IJCTA) 10:885-890

Theron KI, Jacobs G (1996) The effect of irradiance, defoliation, and bulb size on flowering of Nerine bowdenii W. Watson (Amaryllidaceae). J Am Soc Hortic Sci 121:115-122

Thompson DI, Mtshalib NP, Ascough GD, Erwinc JE, Van Staden J (2011) Flowering control in Watsonia: effects of corm size, temperature, photoperiod and irradiance. Sci Hortic 129:493-502

van Ieperen W (2016) Plant growth control by light spectrum: Fact or fiction? Acta Hortic 1134:19-24

Wu PH, Liu CH, Tseng KM, Liu YC, Chen CC, Yang PP, Huang WH, Wang HL (2013) Low irradiance alters carbon metabolism and delays flower stalk development in two orchids. Biol Plant $57: 764-768$

Publisher's Note Springer Nature remains neutral with regard to jurisdictional claims in published maps and institutional affiliations. 